\title{
Radiological features of renal pelvic hemangioma: a case series
}

\author{
Huajun Yu, Weiying Sun, Jianjun Zhang \\ Department of Radiology, Affiliated Zhejiang Hospital, Zhejiang University School of Medicine, Hangzhou, China \\ Contributions: (I) Conception and design: J Zhang; (II) Administrative support: J Zhang; (III) Provision of study materials or patients: H Yu; (IV) \\ Collection and assembly of data: H Yu, W Sun; (V) Data analysis and interpretation: H Yu, W Sun; (VI) Manuscript writing: All authors; (VII) Final \\ approval of manuscript: All authors. \\ Correspondence to: Jianjun Zhang. Department of Radiology, Affiliated Zhejiang Hospital, Zhejiang University School of Medicine, NO. 12 Lingyin \\ Road, Xihu District, Hangzhou 310013, China. Email: 494900594@qq.com.
}

Background: Renal pelvic hemangioma (RPH) is often misdiagnosed as renal pelvis cancer (RPC) due to its similarity in presentation, and there are few reports on the imaging findings of RPH. This study is aimed at improving the understanding of imaging findings specific for RPH by a retrospective analysis of the imaging findings of RPH.

Methods: RPH cases confirmed by pathology and with high-quality images were collected in the analysis. Nine cases of RPH were enrolled, of which 6 cases underwent ultrasound (US); 7 cases underwent computed tomography (CT), including 6 cases with an enhanced scan; and 2 cases underwent magnetic resonance imaging (MRI), including 1 case with an enhanced scan. All images of cases were analyzed and sorted independently by two senior attending radiologist blinded to the pathological results, according to the imaging indicators, such as the density and intensity on CT and MRI respectively. When the opinions between radiologists were inconsistent, images were re-evaluated together until a consensus was reached.

Results: Nine cases of RPH were collected from 5 males and 4 females, aged 16-70 years old, with a median age of 41 years. Five cases were located in the left kidney and 4 cases were located in the right kidney. The clinical symptoms mostly presented with hematuria. Nine cases demonstrated solitary masses, with 4 cases with blurred margins and 5 cases with well-defined margins. The size of the mass was about $1.5-8.0 \mathrm{~cm}$, and the median size was $2.5 \mathrm{~cm}$. The US showed mostly hypoechoic masses and color Doppler flow imaging (CDFI) showed minimal to no blood flow signal. Unenhanced CT scans showed mostly hypodensity and mostly mild continuous enhancement on an enhanced scan. The intensity of lesions was commonly heterogeneous on MRI due to hemorrhage and necrosis. One case showed mild continuous enhancement on an enhanced MRI scan.

Conclusions: The imaging findings of RPH commonly present as a focal lesion with blurred or welldefined margins, mild and continuous enhancement, and no cachexia of the clinical symptoms. RPH should be differentiated from malignant tumors of the renal pelvis for treatment.

Keywords: Hemangioma; renal pelvis; magnetic resonance imaging (MRI); computed tomography (CT); diagnostic imaging

Submitted Jun 01, 2021. Accepted for publication Sep 12, 2021.

doi: $10.21037 /$ tau-21-489

View this article at: https://dx.doi.org/10.21037/tau-21-489

\section{Introduction}

Renal hemangioma is a rare benign tumor of the kidney, mostly located in the renal pelvis (1). Due to the lack of specific clinical and imaging findings, and often accompanied by gross hematuria, renal pelvic hemangioma (RPH) is often misdiagnosed as renal pelvis cancer (RPC) and treated with nephrectomy. In the past, there have been sparsely cases of RPH $(2,3)$, but lack of comprehensive imaging evaluation methods for diagnosis. The cases reported the lesions can 
be manifested as filling defect in the renal pelvis on an excretory urogram, hyperintensity on T1 weighted imaging (T1WI) and hypointensity on T2 weighted imaging (T2WI) with hydronephrosis. Although a definite diagnosis can be confirmed by percutaneous biopsy, the risk of infection or needle tract metastasis is a common clinical concern when the nature of the disease is not clear. Moreover, the treatment and prognosis of $\mathrm{RPH}$ are different from those of RPC. It is necessary to improve the reliability of noninvasive methods for accurate diagnosis, such as radiological techniques for RPH. Radiological techniques play an important role in diagnosis of disease. It can not only observe the size and specific location of the lesion, but also analyze the components of the lesion and provide functional information (4), which is helpful to the localization and qualitative diagnosis of the lesion. Compared with computed tomography (CT) and magnetic resonance imaging (MRI), ultrasound (US) has the advantages of being cost effective, convenient and containing no ionizing radiation. These methods also can be used in the evaluation of treatment and prognosis. Currently, there are only limited literature reports on the radiological findings of RPH, with most of them as case reports $(2,3)$. Therefore, this study sampled 9 cases of RPH with relative complete imaging data in our hospital, and retrospectively analyzed their US, CT, and MRI characteristics.

\section{Methods}

\section{Study participants}

The study was conducted in accordance with the Declaration of Helsinki (as revised in 2013). The study was approved by institutional ethics committee of Affiliated Zhejiang Hospital, Zhejiang University School of Medicine (NO.: 2018-13K) and individual consent for this retrospective analysis was waived. Patients with pathological results and high-quality images of US, CT, or MRI were retrospectively collected. Nine patients were enrolled in our hospital and were pathologically diagnosed as RPH after resection or biopsy from 2005 to 2020. Six cases underwent conventional US examination; 7 cases underwent CT, including 6 cases with an enhanced scan; 2 cases underwent MRI, including 1 case with an enhanced scan.

\section{Image acquisition and analysis}

Due to the long duration over which the study collected data, the technique of US, CT, and MRI were different after an upgrade of the hospital's equipment. However, the scanning methods were similar, such as traditional gray-scale US, three-phase enhancement (arterial phase, venous phase and delayed phase). All US, CT, and MRI images have been deidentified patient specific information, were retrospectively analyzed and summarized by a senior attending radiologist who was blinded to the final diagnosis. Another senior attending radiologist assessed the images again in the same way. When the analytic results were inconsistent, images were re-evaluated together until a consensus was reached. Indicators such as tumor margin, shape, echo density, intensity, and the degree of enhancement after enhanced scan were all collected. Compared to the renal parenchyma, the degree of enhancement on enhanced scan was divided into hypoenhancement, isoenhancement and hyperenhancement.

\section{Statistical analysis}

The measurement data involved in this study were calculated by SPSS 22.0 software (IBM Corp., Armonk, NY, USA), such as age of patients and size of lesions. This study did not utilize statistical tests due to the observational and summative nature of this study.

\section{Results}

The clinical data and imaging findings were summarized in Table 1. A total of 9 cases of RPH were collected in this study, including 5 males and 4 females; age range from 16-70 years old with a median age of 41 years. Five cases were located in the left kidney and 4 cases were located in the right kidney. One case was found following physical examination and 8 cases were found due to hematuria $(8 / 9,88.9 \%)$, including 7 cases of gross hematuria and 1 case of occult blood in the urine (case 3). Four cases were accompanied by flank pain or discomfort. Laboratory and physical examinations are not specific, and the most common ones are hematuria and back tenderness, respectively. All nine cases were of solitary masses, with 4 cases having blurred margins and 5 cases with well-defined margins. The size of the masses was between $1.5-8.0 \mathrm{~cm}$, and the median size was $2.5 \mathrm{~cm}$. US was performed in 6 cases, with 5 cases $(5 / 6,83.3 \%)$ showing a hypoechoic mass, 1 case showing heterogeneous echogenicity, and one showing a cystic component. Color Doppler Flow Imaging (CDFI) showed 4 cases with minimal to no blood flow signal. CT was performed in 7 cases 


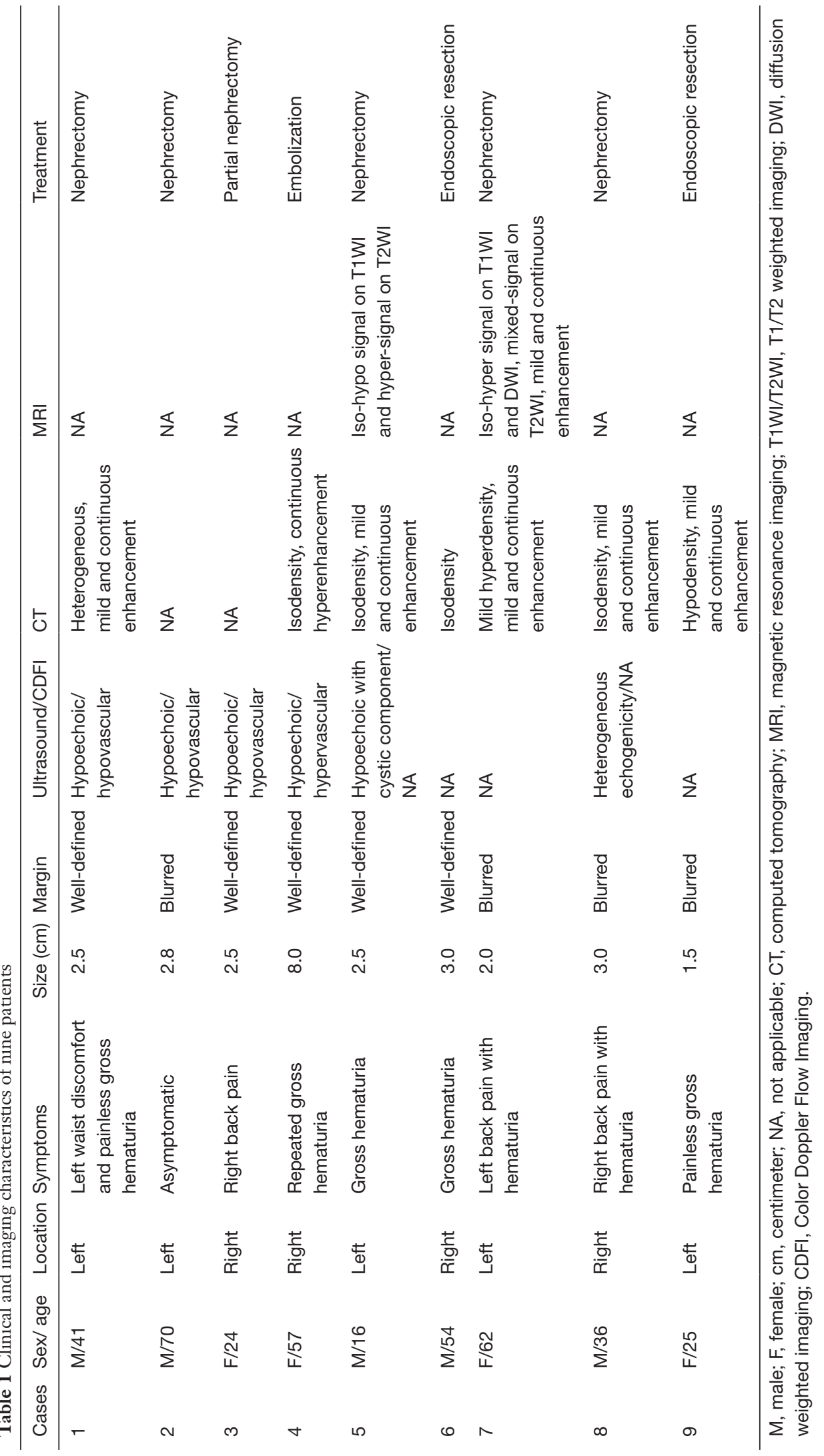



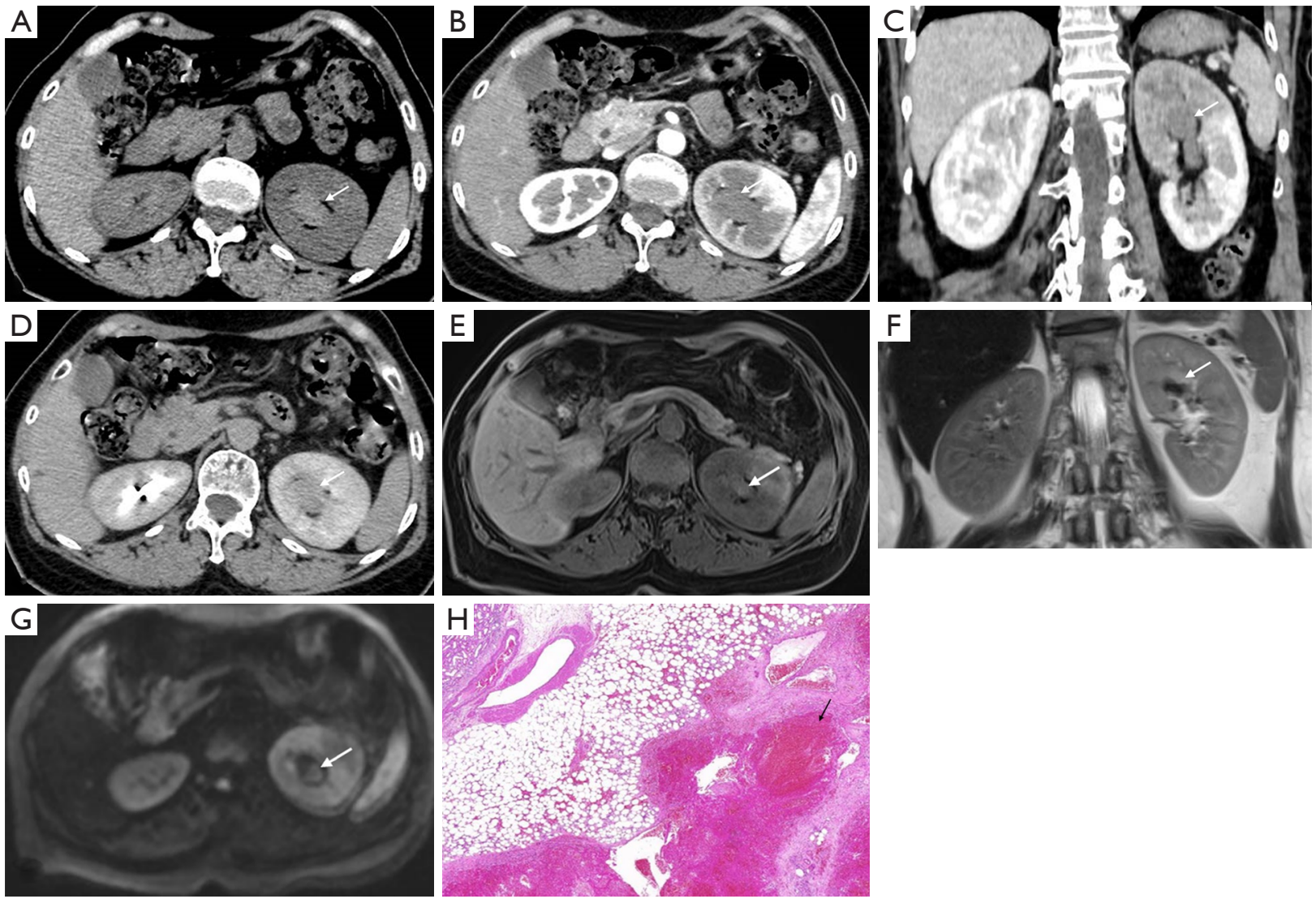

Figure 1 Case 7, a 62-year-old female with left renal pelvic hemangioma. Unenhanced CT showed mild hyperdensity (A). Enhanced CT scan showed mild enhancement in arterial phase (B) and continuous enhancement in venous (C) and delayed phase (D). T1WI showed isohyperintensity (E), T2WI showed mixed intensity with hyper- and hypointensity (F), DWI showed mild hyperintensity (G). Pathology showed vascular hyperplasia with dilation, congestion, bleeding (black arrow), and necrosis (H, hematoxylin-eosin, original magnification $\times 200$, scale bars $=1,000 \mu \mathrm{m})$. The arrows point to the lesion. CT, computed tomography; T1WI, T1 weighted imaging; T2WI, T2 weighted imaging; DWI, diffusion weighed imaging.

including 6 cases with an additional enhanced CT scan. Four cases showed isodensity, 1 case showed hypodensity, 1 case showed mild hyperdensity and 1 case showed mixed density on unenhanced CT scan. Five out of $6(5 / 6$, $83.3 \%$ ) cases showed mild and continuous enhancement on delayed phase (Figure 1 and Figure 2), and 1 out of 6 cases showed hyperenhancement on enhanced CT scan. Two cases underwent MRI included one case underwent an enhanced MRI scan. One case showed iso-hypointensity on T1WI and hyperintensity on T2WI. Another case showed iso-hyperintensity on T1WI and diffusion weighed imaging (DWI), heterogeneous intensity with hyper and hypointensity on T2WI, mild and continuous enhancement on delayed phase of enhanced MRI scan (Figure 1). Two cases showed hydronephrosis in this group. The treatment of 9 patients included 5 cases of nephrectomy, 2 cases of endoscopic resection, 1 case of partial nephrectomy, and 1 case of hemangioma embolization.

\section{Discussion}

Hemangioma is a benign tumor of vascular origin. It is most likely to occur in unipotent vascular cells that have not developed into normal blood vessels during the embryonic period. It is more common in the skin, mucous membranes, and liver, rather than the kidneys. Renal hemangioma is a relatively rare benign tumor of the kidney. About 200 cases have been reported in the previous studies and are commonly localized in the renal pelvis (1). Pathological types of RPH include cavernous, capillary, and fibrous hemangioma. 

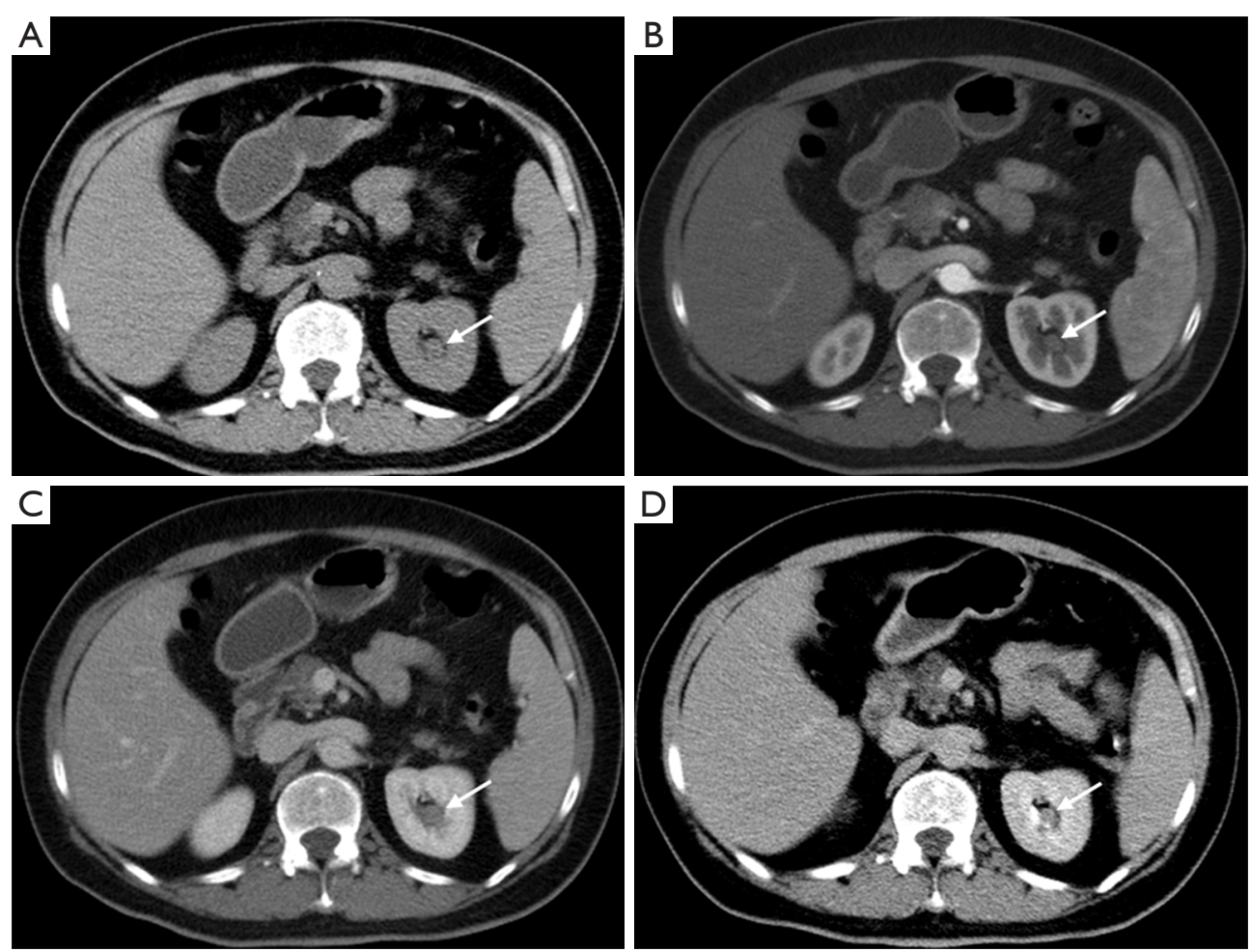

Figure 2 Case 9, a 25-year-old female with left renal pelvic hemangioma. Unenhanced CT showed isodensity (A). Enhanced CT scan showed mild enhancement in arterial phase (B) and continuous enhancement in venous (C) and delayed phase (D). The white arrows point to the lesion. CT, computed tomography.

They typically present with no capsule with a large spongy segment or filled with blood. It is composed of a vascular cavity separated by a small amount of connective tissue $(5,6)$. The clinical manifestations of RPH are mostly presents intermittent gross hematuria, and some patients present with flank pain. Although laboratory and physical examinations lack specificity, they are usually not accompanied by cachexia, which is different from malignant tumors. There is no obvious gender bias for the disorder and masses range from 1-2 cm in diameter (7). The clinical characteristics of this group of cases are consistent with these findings. The diagnosis of RPH needs to be combined with imaging examinations. However, studies describing the imaging characteristics of RPH are limited at case reports. Due to the similarity of clinical and radiographic signs between RPH and RPC, misdiagnosis is common, resulting in unnecessary nephrectomy. The treatment of RPH mainly includes tumor resection, super-selective arterial embolization, and transureteroscopy or ablation rather than nephrectomy. There is no report of postoperative recurrence $(8,9)$. As the treatment differs greatly between RPH and RPC, a greater understanding of the imaging features between the two diagnoses is of great clinical utility.

The imaging findings of RPH: based on previous case reports combined with the imaging findings of this group are as follows: $\mathrm{RPH}$ is usually no more than $3 \mathrm{~cm}$, mostly a unilateral, single mass without obvious calcification, a few may demonstrate cystic components, and the lesion can cause obstructive hydronephrosis. Additional imaging features are as follows: (I) US showed mostly hypoechoic, CDFI mostly showed minimal to no blood flow signal; (II) CT mostly showed iso-hypodensity on nonenhanced scan. Enhanced CT mostly showed mild and continuous enhancement and the surrounding structure was compressed and displaced; (III) MRI commonly showed heterogeneous intensity on T1WI, T2WI, and DWI, due to hemorrhage and necrosis, and mild and continuous enhancement after an enhanced scan. When the lesion is large, it can be manifested as hypervascular and hyperenhancement. The delayed phase on enhanced CT/MRI scan is important for the diagnosis of renal pelvic mass.

Differential diagnosis of RPH is as follows: (I) RPC: Is 
usually multifocal and may involve any part of the collecting system. The tumor can extend to the renal parenchyma, showing a swelling mass, irregular shape, papillary or cauliflower-like distribution and homogeneous density. T1WI will demonstrate isointensity, T2WI will demonstrate hypointensity filling defects due to the hyperintensity of the collection system. The enhanced scan will show mild to moderate homogeneous enhancement (10); (II) Hypovascular renal angiomyolipoma: is common in women but hemorrhage, necrosis and cystic degeneration are rare. The tumor density is homogeneous. The fat suppression on T2WI and T2WI will show hypointensity, which is different from RPH. The enhanced scan will show homogeneous delayed enhancement. The typical sign is the 'cup mouth sign' (10); (III) When RPH occurs in children, it needs to be differentiated from Wilms tumor. Wilms tumor accounts for $87 \%$ of pediatric renal tumors. Wilms tumors are usually larger, but are separated from the residual kidney, accompanied by necrosis, hemorrhage, and cystic degeneration. US and CT/MRI mostly showed heterogeneous echo, density and intensity. T1WI will show iso-hypointensity, while T2WI will show iso-hyper or hypointensity. The enhanced scan will show heterogeneous enhancement $(11,12)$.

The study has several limitations. The small samples were obtained from a single center. Further, the cases collected spanned over a long period of time with different imaging equipment due to the rarity of RPH. Finally, image evaluation and ultrasonic image acquisition present some inherent subjectivity.

\section{Conclusions}

In summary, the imaging findings of RPH commonly present a focal lesion with blurred or well-defined margins, mild and continuous enhancement. The clinical symptoms are diverse but typically present with hematuria and without clinical cachexia. When these findings are present, the possibility of RPH could be considered and should be differentiated from malignant tumors of the renal pelvis. While the diagnosis of RPH using US, CT, or MRI is helpful, the gold standard for diagnosis still relies on immunohistochemical analysis.

\section{Acknowledgments}

We thank Prof. Xiaobin Xu for his assistant for summarizing the results of cases and Michael C. Lin for technical support.
Funding: This work was supported by the medical and health science and technology project of Zhejiang Province [grant numbers 2018KY199 and 2020KY385].

\section{Footnote}

Data Sharing Statement: Available at https://dx.doi. org/10.21037/tau-21-489

Conflicts of Interest: All authors have completed the ICMJE uniform disclosure form (available at https://dx.doi. org/10.21037/tau-21-489). The authors have no conflicts of interest to declare.

Etbical Statement: The authors are accountable for all aspects of the work in ensuring that questions related to the accuracy or integrity of any part of the work are appropriately investigated and resolved. The study was conducted in accordance with the Declaration of Helsinki (as revised in 2013). The study was approved by institutional ethics committee of Affiliated Zhejiang Hospital, Zhejiang University School of Medicine (NO.: 2018-13K) and individual consent for this retrospective analysis was waived.

Open Access Statement: This is an Open Access article distributed in accordance with the Creative Commons Attribution-NonCommercial-NoDerivs 4.0 International License (CC BY-NC-ND 4.0), which permits the noncommercial replication and distribution of the article with the strict proviso that no changes or edits are made and the original work is properly cited (including links to both the formal publication through the relevant DOI and the license). See: https://creativecommons.org/licenses/by-nc-nd/4.0/.

\section{References}

1. Mehta V, Ananthanarayanan V, Antic T, et al. Primary benign vascular tumors and tumorlike lesions of the kidney: a clinicopathologic analysis of 15 cases. Virchows Arch 2012;461:669-76.

2. Motley RC, Patterson DE, Weiland LH. Ureteroscopic visualization of a cavernous hemangioma of the renal pelvis. J Urol 1990;143:788-90.

3. He M, Li M, Sun N, et al. Renal Pelvic Hemangioma in a 3-Year-Old Boy: A Rare Pediatric Case Report. Urology 2018;120:231-3.

4. Grenier N, Quaia E, Prasad PV, et al. Radiology imaging of renal structure and function by computed tomography, 
magnetic resonance imaging, and ultrasound. Semin Nucl Med 2011;41:45-60.

5. Gupta NP, Kumar P, Goel R, et al. Renal sinus hemangioma simulating renal mass: a diagnostic challenge. Int Urol Nephrol 2004;36:485-7.

6. Haas CA, Resnick MI, Abdul-Karim FW. Cavernous hemangioma presenting as a renal hilar mass. J Urol 1998;160:2139-40.

7. Chen T, Jin P, Wang Y, et al. Renal cavernous hemangioma in a child: case report and review of the literature. Urology 2011;78:1185-6.

8. Schlomer BJ. Gross Hematuria in Infancy: A Case of Renal Hemangioma Managed Endoscopically. Urology

Cite this article as: Yu H, Sun W, Zhang J. Radiological features of renal pelvic hemangioma: a case series. Transl Androl Urol 2021;10(10):3766-3772. doi: 10.21037/tau-21-489
2017;101:166-8.

9. Lang EK, Atug F, Thomas R. Selective embolization of capillary hemangioma of the renal papilla. J Urol 2007;177:1146.

10. Pedrosa I, Sun MR, Spencer M, et al. MR imaging of renal masses: correlation with findings at surgery and pathologic analysis. Radiographics 2008;28:985-1003.

11. Lowe LH, Isuani BH, Heller RM, et al. Pediatric renal masses: Wilms tumor and beyond. Radiographics 2000;20:1585-603.

12. Siegel MJ, Chung EM. Wilms' tumor and other pediatric renal masses. Magn Reson Imaging Clin N Am 2008;16:479-97, vi. 